\title{
Long term outcome of twin-twin transfusion syndrome
}

\author{
R B Cincotta, P H Gray, G Phythian, Y M Rogers, F Y Chan
}

\begin{abstract}
Aims-To compare the perinatal mortality and morbidity of infants with twintwin transfusion syndrome (TTTS) with those of gestation matched twin controls and to assess the neurodevelopmental outcome of surviving twins with TTTS. Methods-A cohort of 17 consecutive pregnancies with TTTS was enrolled over three years together with gestation matched twin pregnancies unaffected by TTTS. Serial amnioreduction for the TTTS pregnancies was performed as appropriate. Perinatal death and neonatal morbidities were recorded for both the TTTS cohort and controls. The TTTS survivors had neurodevelopmental follow up to at least 2 years of age.
\end{abstract}

Results-In 12 of the pregnancies, serial amniocenteses were performed, but, in five, the infants were born before intervention. The mean gestational age at delivery was 29.1 weeks (range 23-36). There were five intrauterine deaths in the TTTS cohort and six neonatal deaths (survival 68\%). In the control group, there was one intrauterine death and five neonatal deaths (survival $82 \%$ ). Infants in the TTTS group had a greater requirement for inotropes $(p=0.04)$ and a higher incidence of renal failure $(p=0.005)$. Periventricular leucomalacia and cerebral atrophy were seen in $17 \%$ of the TTTS group, but none of the controls $(p=0.03)$. The 23 surviving TTTS infants were all followed up, with $22 \%$ having significant neurological morbidity: cerebral palsy and global developmental delay.

Conclusions-Twins with TTTS have high perinatal mortality and neonatal morbidity, and long term neurodevelopmental morbidity in survivors is high. Further investigation into the pathogenesis and management of TTTS is required.

(Arch Dis Child Fetal Neonatal Ed 2000;83:F171-F176)

Keywords: twin-twin transfusion syndrome; amnioreduction; morbidity; neurodevelopmental outcome

Development Unit,

Mater Children's

Hospital, Raymond

Terrace, South

Brisbane, Queensland,

Australia

P H Gray

Y M Rogers

Correspondence to:

Dr Cincotta, Department of

Maternal-Fetal Medicine,

Raymond Terrace, South

Brisbane, Queensland 4101,

Australia

1neoap@mater.org.au

Accepted 3 February 2000 delivery and loss of both twins. Over the past decade, recognition of this condition and its treatment has resulted in improved survival rates. The most commonly used treatment is amnioreduction, where several litres of amniotic fluid are removed in an attempt to prevent premature delivery. However, preterm delivery is still often required because of growth restriction, hydrops, or other complications. Survival rates of $60-80 \%$ are now commonly reported. ${ }^{3-6}$ The other major treatment modality for TTTS is laser ablation of the placental anastomosing vessels, with survival rates of $50-70 \%$ having been reported..$^{7-11}$ Thus twins with TTTS continue to have a high perinatal death rate despite modern therapeutic interventions. Although the twin to twin transfusion process results in a high rate of death in utero, its contribution to neonatal mortality and morbidity, in addition to that from prematurity alone, remains unclear.

Few studies of TTTS have reported neonatal morbidities in detail, although the incidence of cerebral abnormalities detected by cranial ultrasound has been described. ${ }^{52-14}$ Major abnormalities have occurred in 10-29\% of survivors. Cardiac dysfunction has also been reported in twins with TTTS, both in utero and during the neonatal period. ${ }^{51516}$ No specific abnormality has been found on echocardiography, although cardiomegaly and tricuspid regurgitation have been invariably described, together with a number of cases of right heart outflow obstruction. No study, however, has compared the perinatal outcome and neonatal morbidity of twins with TTTS with dichorionic or monochorionic twins without TTTS.

Attention is now focusing on the long term outcomes of TTTS. Early reports suggested that survivors of TTTS treated with amnioreduction had a high incidence of adverse neurological outcomes, with the incidence of cerebral palsy being quoted as high as $25 \% .^{4}{ }^{68}$ In contrast, rates of cerebral palsy reported in more recent studies after treatment with laser ablation are in the region of $5 \% .{ }^{911}$ Although there is more information on short term outcomes and complications of TTTS, there are few data on how neonatal findings correlate with long term consequences of TTTS.

The aim of this study is to compare the short term perinatal morbidity and mortality of twins with TTTS managed by antenatal amnioreduction with those of gestation matched twin controls. The long term neurodevelopmental outcomes of survivors of TTTS are also documented. 


\section{Methods}

Eighteen consecutive cases of TTTS were identified from the beginning of 1994 until the end of 1996 in the Department of MaternalFetal Medicine of the Mater Mothers' Hospital. TTTS was diagnosed in all cases by the ultrasound criteria of severe polyhydramnios in one twin sac and severe oligohydramnios/ anhydramnios in the co-twin of a monochorionic pregnancy. The twin in the oligohydramniotic sac was in a fixed position and appeared to be "stuck" to the adjacent uterine wall. A dividing membrane between the two sacs was visualised by ultrasonography. All placentas were examined after delivery, and monochorionicity was confirmed. A spontaneous abortion occurred in one case at 18 weeks gestation before any intervention, and this was excluded from further analysis. Serial amnioreduction was the standard treatment during the study period for maternal discomfort and in an attempt to prevent preterm delivery. After delivery, the infants were managed in the neonatal intensive care unit. For the index twin pregnancies, a set of controls were identified from the hospital's database. The control pregnancies were the next twin pregnancy delivered at a matched gestational age $( \pm 1$ week gestation) that was not associated with TTTS. They were not matched for chorionicity, with $18 \%$ of the controls being monochorionic.

Perinatal mortality (intrauterine and neonatal) was compared between the TTTS cases and controls. Neonatal morbidities between the two groups were also compared. A full blood count was performed on the first day of life in all newborn infants. For the purposes of the study, hypotension was defined as a mean blood pressure below that acceptable for gestation and requiring treatment with colloid/ crystalloid and/or inotropic support. Inotropes were only used for hypotension refractory to treatment with colloid/crystalloid. Renal failure was defined as a urine output of $<1 \mathrm{ml} / \mathrm{kg} / \mathrm{h}$ during the first three days of life. Serum creatinine was measured on a daily basis during the first few days of life. Serial cranial ultrasound scans were performed during the first and fourth weeks of life and before discharge as previously described. ${ }^{17}$ Additional scanning was undertaken as clinically indicated. Echocardiography was performed only if clinically indicated.

Neonatal survivors of TTTS were followed up in the multidisciplinary Growth and Devel-

Table 1 Obstetric data and pregnancy outcomes of twins with twin-twin transfusion syndrome (TTTS) and controls

\begin{tabular}{|c|c|c|}
\hline & TTTS & Controls \\
\hline Number of pregnancies & 17 & 17 \\
\hline Gestation at diagnosis (weeks) & $21.8(18-28)$ & $\mathrm{N} / \mathrm{A}$ \\
\hline Gestation at delivery (weeks) & $28.7(23-36)$ & $28.9(23-36)$ \\
\hline Number of amnioreductions & $2.1(1-4)$ & N/A \\
\hline Total volume of amniotic fluid drained (litres) & $4.4(1.6-13.6)$ & $\mathrm{N} / \mathrm{A}$ \\
\hline Intrauterine fetal death & 5 & 1 \\
\hline Neonatal death & 6 & 5 \\
\hline Surviving twins & $(68)$ & $(82)$ \\
\hline Pregnancies with both twins & $10 \quad(59)$ & $(76)$ \\
\hline Pregnancies with one twin & 3 (18) & (12) \\
\hline Pregnancies with neither twin & $4 \quad(24)$ & $(12)$ \\
\hline
\end{tabular}

Values are number, number (\%), or mean (range).

N/A, not applicable. opment Unit of the Mater Children's Hospital; the members of the team were unaware of the TTTS status of the children. Systematic follow up of the control infants was not possible. After a medical history, a detailed neurological examination was performed. Cerebral palsy was diagnosed on the basis of this examination and the following standard criteria: persistent abnormality of movement and posture resulting in impairment of function, caused by a non-progressive lesion of the immature brain. ${ }^{18}$ The children were also seen by a paediatric physiotherapist, and a neurosensory motor developmental assessment was performed. ${ }^{19}$ In all cases, agreement on the diagnosis of cerebral palsy was reached. The psychologist attending the clinic performed a Griffith's developmental assessment. A general quotient (GQ) more than 2 standard deviations below the mean $(\mathrm{GQ}<76)$ represented global developmental delay.

Results of categorical variables were compared using Fisher's exact test. Continuous normally distributed variables were examined with Student's $t$ test. Analysis was performed using Statistix 4.0

\section{Results}

Seventeen sets of twins with TTTS were evaluated. The gestational age at diagnosis ranged from 18 to 28 weeks with a mean (SD) age of 21.5 (3.5) weeks. Twelve pregnancies were treated with serial amnioreduction, with the number of amniocenteses ranging from one to four per patient (mean 2.1). The total volume of fluid drained ranged from 1.6 to 13.6 litres (mean 4.4 litres). In five cases, amnioreduction was not performed, either because of delivery immediately after presentation or because of intrauterine death of one twin. Delivery occurred at a mean (SD) gestational age of 28.7 (3.6) weeks (range 23-36). The mean (SD) gestational age of the controls was 28.9 (4.0) weeks.

Table 1 shows that the intrauterine death rate for the TTS pregnancies was higher than for the controls, although this was not statistically significant $(p=0.10)$. The neonatal death rates for the two groups were almost identical. The overall perinatal survival rate of the TTTS group was $68 \%$ (95\% confidence intervals 50\% to $83 \%$ ) compared with a survival rate of $82 \%$ ( $95 \%$ confidence intervals $66 \%$ to $93 \%$ ) in the controls $(p=0.13)$. In the TTTS group, both twins survived in $59 \%$, one baby died in $18 \%$, and both twins died in $24 \%$ of the pregnancies evaluated. In comparison, $76 \%$ of the twin pregnancies in the control group resulted in both twins surviving. Furthermore, in $12 \%$ of these pregnancies, only one twin survived. The remaining $12 \%$ of pregnancies resulted in no neonatal survivors.

There was no significant difference in the mean birth weights between the TTTS twins $(1127(636) \mathrm{g})$ and the controls (1282 (607) g) $(p=0.30)$, nor between the donor and recipient TTTS twins. When both twins were liveborn, the initial haemoglobin levels were analysed. In the TTTS group, the mean haemoglobin concentration was found to be 
Table 2 Neonatal morbidities of twins with twin-twin transfusion syndrome (TTTS) and controls

\begin{tabular}{lrrrrr}
\hline & \multicolumn{2}{c}{ TTTS $(n=29)$} & \multicolumn{2}{c}{ Controls $(n=33)$} & p Value \\
\hline Transient tachypnoea of the newborn & 9 & $(31 \%)$ & 4 & $(12 \%)$ & 0.06 \\
Respiratory distress syndrome & 13 & $(45 \%)$ & 20 & $(61 \%)$ & 0.16 \\
CNLD & 8 & $(28 \%)$ & 10 & $(30 \%)$ & 0.52 \\
Hypotension & 15 & $(52 \%)$ & 13 & $(39 \%)$ & 0.23 \\
Dopamine administration & 14 & $(48 \%)$ & 8 & $(24 \%)$ & 0.04 \\
Renal failure & 14 & $(48 \%)$ & 5 & $(15 \%)$ & 0.005 \\
Mean (SD) creatinine (mmol/l) & $0.14(0.09)$ & 0.09 & $(0.02)$ & 0.01 \\
Sepsis & 17 & $(59 \%)$ & 13 & $(39 \%)$ & 0.10 \\
Necrotising enterocolitis & 1 & $(3 \%)$ & 0 & & 0.46 \\
Seizures & 3 & $(10 \%)$ & 1 & $(3 \%)$ & 0.25 \\
PVH (grade 1-2) & 2 & $(7 \%)$ & 7 & $(21 \%)$ & 0.41 \\
PVH (grade 3-4) & 0 & \multicolumn{1}{c}{$(10 \%)$} & 1 & $(3 \%)$ & 0.53 \\
Cystic PVL & 3 & $(10 \%)$ & 0 & & 0.09 \\
Cerebral atrophy & 2 & $(7 \%)$ & 0 & & 0.21 \\
\hline
\end{tabular}

CNLD, chronic neonatal lung disease; $\mathrm{PVH}$, periventricular haemorrhage; $\mathrm{PVL}$, periventricular leucomalacia.

lower in the donor twin than the recipient twin (14.8 (3.4) $v 18.2(4.2) \mathrm{g} / \mathrm{dl}, \mathrm{p}=0.02)$. In the control group, there was no difference in the mean haemoglobin level between twin 1 and twin 2 (16.0 (3.1) v 16.4 (2.6) g/dl, p = 0.73).

Table 2 shows the neonatal characteristics of the TTTS twins and the controls. Acute respiratory morbidity, either transient tachypnoea of the newborn or respiratory distress syndrome, occurred with a similar incidence in the two groups, with chronic neonatal lung disease also occurring with similar frequency. Although the incidence of hypotension did not differ between the groups, hypotension requiring inotropic support was more common in the TTTS twins. Oliguric renal failure was more common in the TTTS twins, and the mean maximum creatinine level was also higher. Importantly, renal function returned to normal in all survivors before discharge. There was no difference in the rate of infection, including sepsis and necrotising enterocolitis, between the groups. None of the controls had clinically diagnosed seizures, but this was not statistically significant when compared with the TTTS twins. Haemorrhagic lesions diagnosed by cranial ultrasound occurred more commonly in the control group, but this also was not statistically significant. The one infant with a grade 4 periventricular haemorrhage (PVH) died in the neonatal period.

Cystic periventricular leucomalacia (PVL) was diagnosed in three TTTS infants. One infant had increased periventricular echogenicity detected on an ultrasound scan on day 7 . The echogenicity resolved, but a scan at 2 months disclosed cystic PVL adjacent to the left lateral ventricle. A second infant had normal ultrasound scans during the first weeks of life. A computed tomography (CT) scan performed at 3 months of age because of concerns about the infant's muscle tone showed bilateral periventricular cysts in the parietooccipital regions. The third infant had bilateral cystic PVL in the parieto-occipital region on an ultrasound scan on day 24. Cerebral ultrasonography during the first week of life was normal, while a CT scan on day 11 showed a reduction in white matter density, but no cyst formation. Two infants had evidence of cerebral atrophy. The first infant had a grade 1 PVH on initial scans that resolved on follow up. An ultrasound scan at 3 months of age, however, showed that there was appreciable prominence of the subarachnoid space, which was confirmed on CT scan. The second infant had no PVH, but a follow up ultrasound at 6 weeks of age showed asymmetrical dilatation of the lateral ventricles. The changes seen in both infants were consistent with cerebral atrophy. None of the control group displayed any evidence of cerebral ischaemia, this being statistically significant compared with the TTTS twins $(p=0.03)$. Of note, none of the cerebral lesions were present on the initial ultrasound scans, performed during the first days of life, but became evident during the period of hospitalisation.

Analysis of the TTTS group according to whether the twins were donor or recipient showed no difference in neonatal morbidities. In particular, renal failure occurred in seven of the 14 donor twins and seven of the 15 recipient twins. Ischaemic brain lesions were found in three of the donor twins and two of the recipient twins.

All the survivors of TTTS were seen for follow up in the growth and development clinic at the age of at least 2 years (2-4.5 years) corrected for prematurity. Three infants were diagnosed with cerebral palsy, of whom two also had developmental delay, while two had global developmental delay alone. All of these cases showed neonatal ultrasound abnormalities (table 3). Case 1 was a child with moderately severe cerebral palsy, who also had considerable visual problems and developmental delay. Cases 2 and 3 were from the same twin pregnancy. The former had mild spastic diplegia (walking at the age of 2 years) and also had developmental delay, while her twin had developmental delay alone (GQ 63). In case 4, developmental delay (GQ 50) and also significant visual and auditory impairments were present. Case 5 was a child with spastic quadriplegia. Thus five of the 23 surviving infants (22\%; $95 \%$ confidence intervals $7 \%$ to $44 \%$ )

Table 3 Case descriptions of survivors of twin-twin transfusion syndrome (TTTS) with adverse neurodevelopmental outcomes

\begin{tabular}{|c|c|c|c|c|c|c|c|}
\hline $\begin{array}{l}\text { Case } \\
\text { number }\end{array}$ & Twin & $\begin{array}{l}\text { Gestation at } \\
\text { delivery }\end{array}$ & Amnioreduction & $\begin{array}{l}\text { Neonatal ultrasound } \\
\text { changes }\end{array}$ & Outcome & Outcome of other twin & Comments \\
\hline 1 & Donor & 29 & 1 & Cerebral atrophy & $\mathrm{CP}, \mathrm{GDD}$ & Survived normal & $\begin{array}{l}\text { Initially, recipient twin was hydropic at } 23 \\
\text { weeks and then resolved. At } 29 \text { weeks the } \\
\text { donor became acutely hydropic, while the } \\
\text { recipient became bradycardic. Urgent } \\
\text { caesarean section }\end{array}$ \\
\hline 2 & Donor & 28 & 0 & PVL & CP, GDD & GDD & \\
\hline 3 & Recipient & 28 & 0 & Cerebral atrophy & GDD & $\mathrm{CP}$ & \\
\hline 4 & Donor & 27 & 3 & PVL & GDD & Survived normal & \\
\hline 5 & Recipient & 33 & 3 & PVL & $\mathrm{CP}$ & IUFD & Delivered $24 \mathrm{~h}$ after IUFD \\
\hline
\end{tabular}

PVL, periventricular leucomalacia; CP, cerebral palsy; GDD, global developmental delay; IUFD, intrauterine fetal death. 
had neurodevelopmental disability, with perinatal death or neurological morbidity occurring in 16 of $34(47 \% ; 95 \%$ confidence intervals $30 \%$ to $65 \%$ ) TTTS twins. It is notable that all these infants had abnormal brain scans, and furthermore all with cerebral lesions indicative of cerebral ischaemia developed neurodevelopmental problems.

\section{Discussion}

TTTS is associated with a high perinatal death rate which, without treatment, approaches $100 \%{ }^{20}$ With the use of serial amnioreduction, the perinatal survival in our series of 17 pregnancies was $68 \%$, which is very similar to that found in other reports using this technique for management. ${ }^{4122}$ The perinatal survival of TTTS pregnancies after treatment with laser ablation of placental vascular anastomoses has improved from $50 \%{ }^{78}$ to $60-70 \% .^{1011{ }^{14}}$ Unfortunately, there is still a significant risk of fetal loss immediately after the procedure. Accordingly the overall perinatal mortality for TTTS twins remains high despite intervention. Although there was a higher rate of intrauterine fetal death in the TTTS twins than in the controls, the same was not found for neonatal death. This suggests that, although the twin to twin transfusion process places the fetus at risk in utero, the degree of prematurity poses the greater risk for the newborn infant. A larger cohort needs to be studied together with controls to examine this further.

Analysis of the types of neonatal morbidities in our series suggests that the infants with TTTS do suffer from more organ specific problems. Acute renal failure diagnosed on the basis of reduced urinary output and high creatinine levels occurred more often in the twins with TTTS than the controls. Perhaps surprisingly, renal failure was present as commonly in the recipient (polyhydramniotic) twin as the donor (oligohydramniotic) twin. This differs from the report of Cincotta et $a l,{ }^{5}$ who found that renal failure only occurred in donor twins, but is in accordance with Reisner et $a l,{ }^{4}$ who found that renal failure was also present in some of the recipient twins. Overall, the findings would suggest that twins with TTTS, being delivered extremely prematurely, have significant cardiorespiratory morbidity, and thus recipient twins are as likely to develop acute renal failure as donors. Long term renal impairment, however, does not appear to be a problem. Echocardiography during the neonatal period was only performed when clinically indicated, mainly for a suspected patent ductus arteriosus, and thus no overall judgment on cardiac function could be made. Hypotension, however, of such severity that inotropic support was required was found more commonly in the TTTS group than in the controls. This indeed would suggest that significant cardiac dysfunction was present in many infants as described by others. ${ }^{16}$ These infants should therefore have routine assessment of cardiac function to enable optimal management.

There was a significant number of abnormal cranial ultrasound findings in survivors of TTTS compared with controls. All infants in our series had serial cranial ultrasonography. Minor PVH was found in both the infants with TTTS and the controls, but these lesions were unlikely to result in long term neurodevelopmental morbidity. The one control infant with a grade 4 haemorrhage died in the neonatal period. Injury to cerebral white matter is more likely to have long term consequences. Three of our TTTS survivors had cystic PVL, with a further two having changes on scan consistent with cerebral atrophy. Similar findings have also been reported by Cincotta et al, ${ }^{5}$, with cerebral atrophy also being described by Trespidi et al. ${ }^{6}$ Mari $^{12}$ reported results from an international multicentred registry of amnioreduction for TTTS and found that $20 \%$ had major abnormalities on cranial ultrasound. Dickinson and Evans, ${ }^{23}$ reporting the results from another multicentred registry, found an incidence of $28 \%$ abnormal cranial ultrasounds including $11 \%$ with PVL.

Denbow et al, ${ }^{13}$ however, found that $35 \%$ of infants with TTTS had evidence of an antenatal cerebral ischaemic insult, with lesions including white matter cysts, basal ganglia echogenicity, and mild lateral ventricular dilatation. The significance of these findings is unclear, as no long term outcomes were described. We did not find evidence of white matter damage on early scans, with the lesions only becoming evident on follow up. Although this may suggest that the cerebral injury in our infants occurred postnatally, a recent report using diffusion weighted magnetic resonance imaging techniques ${ }^{24}$ found evidence of cerebral ischaemia at a time when the ultrasound scan was normal. Thus it is possible that the cystic PVL seen in the present study had its origins antenatally. The cerebral atrophy that was noted in two infants evolved over a number of months. Both infants had significant complications during the neonatal period, including severe chronic lung disease. Accordingly it would not be possible to determine whether the cerebral insult in theses infants occurred antenatally or as a consequence of postnatal events.

Relatively few reports have included the long term outcomes of TTTS twins. Initial studies have suggested an incidence of cerebral palsy of up to $25 \%,{ }^{425}$ when the condition was managed by amnioreduction. Trespidi et $a l,{ }^{6}$ who pursued a policy of aggressive amnioreduction, did not document mode or duration of follow up, but found that $15 \%$ had severe handicaps. All our surviving infants were seen for systematic follow up at our multidisciplinary growth and development clinic to at least 2 years of age corrected for prematurity, with $13 \%$ of survivors having cerebral palsy and a further $8 \%$ having global developmental delay. We were unable to follow up the gestation matched twin controls, so comparisons of the rate of disability are not possible. In a follow up study of infants born at 24-29 weeks gestation at our centre, however, the rate of cerebral palsy and global developmental delay was $8 \%,{ }^{26}$ suggesting that the twins with TTTS have a high rate of neurodevelopmental morbidity. 
The reports of neurological outcome of TTTS twins after treatment with laser ablation of placental anastomoses suggest a lower incidence of cerebral palsy, with most series suggesting an incidence no greater than $5 \% .^{911}$ In the study of De Lia et al, ${ }^{11}$ the mean duration of follow up was 14 months, with the outcome being available to just 1 month in some infants. Accordingly it is possible that there was an under-representation of the true incidence of cerebral palsy in their study. Ville et a ${ }^{9}$ reported cerebral palsy or suspected neurological handicap in only $4.2 \%$ of survivors at the age of at least 1 year. Of note, however, almost $70 \%$ of their deliveries occurred at 32 weeks gestation or greater. This compares with a mean gestational age at delivery in our series of 28.7 weeks. Furthermore, this result has to be taken in the context of a perinatal survival of $55 \%$, in comparison with a survival of $68 \%$ in the present study.

Laser ablation of placental vessels aims to interrupt the vascular communications between the circulations of the two fetuses, although there is a resultant significant risk of intrauterine death. When this occurs in the donor twin, it seems that this is a consequence of acute obliteration of part of the placenta of a fetus who is already compromised. When both twins die in utero, this may be the result of incomplete coagulation of anastomosing vessels. Instead of alleviating the shunting between the fetuses, the situation is made worse and hence hastens their demise. ${ }^{9}$ If the laser coagulation is successful, however, the pregnancy should be prolonged and there will be interruption of the haemodynamic events which predispose the twins to visceral damage including cerebral injury, ${ }^{27}$ which may account for the relatively low reported incidence of neurodevelopmental disabilities. With improvements to the laser technique, however, it may be possible to decrease the procedure related loss rate. Serial amnioreduction on the other hand, by alleviating the severe polyhydramnios, aims to result in the prolongation of a TTTS pregnancy. The underlying pathology of shunting between the fetuses through anastomotic vessels, however, is unchanged. Hecher et $a l^{14}$ compared endoscopic laser surgery and serial amniocenteses in the treatment of severe TTTS by analysing the results of two European centres. In the laser surgery group, there were significantly more pregnancies with the intrauterine death of one fetus, but fewer with the intrauterine death of both fetuses. Overall, the survival was similar in the two groups. Importantly, even though they did follow up survivors, there was a higher incidence of abnormal brain scans (PVL, grade 3 and 4 PVH, parenchymal defects, and microcephaly) in the amniocentesis group. Thus, although treatment of TTTS by either serial amniocenteses or laser treatment has advocates, the only certain way of comparing the efficacy of such treatments is by a randomised controlled clinical trial. Thus the results of the Eurofetus Trial (www.eurofetus.org) comparing the outcomes of laser ablation and amnioreduc- tion for the treatment of TTTS are eagerly awaited.

TTTS is associated with high perinatal mortality and morbidity as well as the likelihood of prematurity. There is a significant risk of cerebral palsy and neurodevelopmental delay. Our study confirms the high incidence of these complications in the survivors of TTTS. This information is important in counselling women with TTTS. It is also important to focus attention on areas where research may improve our understanding of the mechanisms of this condition and also lead to the development of improved treatments.

We gratefully acknowledge the assistance of the Growth And Development Unit team for their invaluable assistance with the follow up assessments.

1 Cincotta RB, Fisk NM. Current thoughts on twin-twin ransfusion syndrome. Clin Obstet Gynaecol 1997;40;290 302.

2 Sebire NJ, D'Ercole C, Carvelho M, Sepulveda W, Nicolaides KH. Inter-twin membrane folding in monochorionic pregnancies. Ultrasound Obstet Gynecol 1998; 11:324-7

3 Elliott JP, Urig MA, Clewell WH. Aggressive therapeutic amniocentesis for treatment of twin-twin transfusion syndrome. Obstet Gynaecol 1991;77:537-40.

4 Reisner DP, Mahony BS, Petty CN, et al. Stuck twin syndrome: outcome in thirty-seven consecutive cases. $\mathrm{Am}$ f Obstet Gynecol 1993;169:991-5.

5 Cincotta R, Oldham J, Sampson A. Antepartum and postpartum complications of twin-twin transfusion. Aust NZ7 Obstet Gynaecol 1996;36:303-8.

6 Trespidi L, Boschetto C, Caravelli E, Villa L, Kustermann A, Nicolini U. Serial amniocenteses in the management of twin-twin transfusion syndrome; when is it valuable? Fetal Diagn Ther 1997;12:15-20.

7 De Lia JE, Kuhlmann RS, Harstad TW, et al. Fetoscopic laser ablation of placental vessels in severe previable twintwin transfusion syndrome. Am F Obstet Gynecol 1995; 172:1202-11.

8 Ville Y, Hyett J, Hecher K, Nicolaides K. Preliminary experience with endoscopic laser surgery for severe twin-twin transfusion syndrome. N Engl f Med 1995; 332:224-7.

9 Ville Y, Hecher K, Gagnon A, Sebire N, Hyett J, Nicolaides $K$. Endoscopic laser coagulation in the management of severe twin-to-twin transfusion syndrome. Br f Obstet Gynaecol 1998;105:446-53.

10 Quintero RA, Morales WJ, Mendoza G, et al. Selective photocoagulation of placental vessels in twin-twin transfusion syndrome: evoluation of a surgical technique. Obstet Gynecol Surv 1998;53(suppl 12):S97-103.

11 De Lia JE, Kuhlmann RS, Lopez KP. Treating previable twin-twin transfusion syndrome with fetoscopic laser surgery: outcomes following the learning curve. $\mathcal{f}$ Perinat Med 1999;27:61-7.

12 Mari G. Amnioreduction in twin-twin transfusion syndrome: a multicenter registry of 579 procedures. $A m \mathcal{F}$ Obstet Gynecol 1998;178:S28

13 Denbow ML, Battin MR, Cowan F, Azzopardi D, Edwards $\mathrm{AD}$, Fisk NM. Neonatal cranial ultrasonographic findings in preterm twins complicated by severe fetofetal transfusion syndrome. Am $\mathcal{F}$ Obstet Gynecol 1998;178: 479-83.

14 Hecher K, Plath H, Bregenzer T, Hansmann M, Hackelöer BJ. Endoscopic laser surgery versus serial amniocenteses in the treatment of severe twin-twin transfusion syndrome. Am F Obstet Gynecol 1999;180:717-24.

15 Zosmer N, Bajora R, Weiner E, Ribby M, Vaughan J, Fisk NM. Clinical and echographic features of in utero cardiac dysfunction in the recipient twin in twin-twin transfusion syndrome. Br Heart 7 1994; 72:74-9.

16 Simpson LL, Marx GR, Elkadry EA, D'Alton ME. Cardiac dysfunction in twin-twin transfusion syndrome: a prospective study. Obstet Gynecol 1998;92:557-62

17 Tudehope DI, Masel J, Mohay H, et al. Neonatal cranial ultrasonography as predictor of 2 year outcome of very low birthweight infants. Australian Paediatric fournal 1989; 25:66-71.

8 Bax MCO. Terminology and classification of cerebral palsy. Dev Med Child Neurol 1964;6:295-307.

19 Burns YR. NSMDA. Physiotherapy assessment for infants and young children. Brisbane: Copyright Publishing Company, 1992 .

20 Weir PE, Ratten GJ, Beischer NA. Acute polyhydramnios: a complication of monozygous twin pregnancy $\mathrm{Br} \mathcal{F}$ Obstet Gynaecol 1979;86:849-53.

21 Pinette MG, Pan Y, Pinette SG, Stubblefield PG. Treatment of twin-twin transfusion syndrome. Obstet Gynecol 1993;82:841-6. 
22 Dennis LG, Winkler CL. Twin-to-twin transfusion syndrome: aggressive therapeutic amniocentesis. Am $\mathcal{F}$ Obstet Gymecol 1997;177:342-9.

23 Dickinson JE, Evans SF. Obstetric and perinatal outcomes from the Australian and New Zealand Twin-Twin Syndrome Registry. Am f Obstet Gynecol 2000;182:706-12.

24 Inder T, Huppi PS, Zientara GP, et al. Early detection of periventricular leukomalacia by diffusion-weighted magnetic resonance imaging techniques. F Pediatr 1999; 134:631-4.
25 Ville Y, Hyett J, Nicolaides K. Twin-twin transfusion. N Engl f Med 1995;333:388-9.

26 Gray PH, Hurley TM, Rogers YM, et al. Survival and neonatal and neurodevelopmental outcome of 24-29 week gestation infants according to primary cause of preterm delivery. Aust N Z F Obstet Gynaecol 1997; 37:161-8.

27 Rehan VK, Menticoglou SM. Mechanism of visceral damage in fetofetal transfusion syndrome. Arch Dis Child 1995;73:F48-50. 